\title{
A comparative study of the linear and colour-flavour-locked equation of states for compact objects
}

\author{
S. Thirukkanesh ${ }^{1, \mathrm{a}}$, Anand Kaisavelu ${ }^{2, \mathrm{~b}}$, Megan Govender ${ }^{3, \mathrm{c}}$ \\ ${ }^{1}$ Department of Mathematics, Faculty of Science, Eastern University, Vantharumoolai, Chenkalady 30350, Sri Lanka \\ 2 Astrophysics and Cosmology Research Unit, School of Mathematics, Statistics and Computer Science, University of KwaZulu-Natal, \\ Private Bag X54001, Durban 4000, South Africa \\ ${ }^{3}$ Department of Mathematics, Faculty of Applied Sciences, Durban University of Technology, Durban 4000, South Africa
}

Received: 30 December 2019 / Accepted: 22 February 2020 / Published online: 6 March 2020

(C) The Author(s) 2020

\begin{abstract}
In this work we present a general framework for obtaining exact solutions to the Einstein field equations describing strange stars obeying a colour-flavour-locked (CFL) equation of state. Starting off with a spherically symmetric metric in isotropic coordinates describing the interior of the star, we impose a CFL equation of state to reduce the problem to a single-generating function of the gravitational potentials. Our approach leads to an infinite class of solutions of the field equations. In order to test the physical viability of our solutions, we subscribe a particular model to stringent stability tests. In particular, we show that a linear equation of state described by the MIT Bag model mimics the CFL equation of state describing strange stars with interacting quark matter. This is an interesting result which connects the more robust and mathematically tractable linear equation of state to the fundamental physics describing nuclear matter in the quark regime.
\end{abstract}

\section{Introduction}

Compact objects such as neutron stars and pulsars are natural laboratories for testing our understanding of fundamental interactions arising in particle physics. There has been huge strides in explaining the physical attributes such as compactness, radii, redshifts and luminosities of these objects based on microphysics. This interplay between the microphysics and the macrophysics (quite literally on an astronomical scale) has come to the fore quite frequently in the recent past. Delgaty and Lake constructed a catalogue containing all analytic solutions that describe isolated static spherically

\footnotetext{
a e-mail: Thirukkanesh@esn.ac.lk

b e-mail: Anand.Kaisavelu@gmail.com

c e-mail: MegandhrenG@dut.ac.za (corresponding author)
}

symmetric perfect fluid solutions [1]. It is interesting to note that out of the 127 solutions scrutinised, a mere 16 are physically viable in their description of a bounded stellar configuration. Furthermore, a paltry 9 solutions obey the causality condition. Despite the consistency of this small number of relevant solutions describing stellar objects there is very little or no connection to the microphysics governing the fluid composition.

A fruitful and physically plausible approach to stellar modeling within the framework of classical general relativity (CGR) is to adopt an equation of state (EoS) a priori. The EoS intimately connects the matter variables, for example, the energy density and the pressure of the stellar fluid [24]. In the past the natural and simplest EoS related the fluid density and pressure via an ad-hoc linear relation. The MIT Bag model motivated by work in QCD centred on quark confinement and asymptotic freedom [6] is essentially a linear EoS of the form $p=(\rho-4 B) / 3$, where $B$ is the Bag constant $[7,8]$. The MIT Bag model has been employed in several recent works to generate models of anisotropic spheres $[9,10]$. As pointed out by [11], this EoS cannot adequately account for the deconfinement of quarks at high density. Furthermore, the magnitude of the Bag constant is not absolute but rather depends on the compact objects being modeled [12]. An interesting study of compact objects in CGR using the Vaidya-Tikekar ansatz revealed a linear relationship between the energy density and the isotropic pressure. In this work, the gravitational behaviour and pressure isotropy were specified at the outset. The resulting solution when tested for physical viability revealed the presence of a linear EoS.

Polytropes and their stability have been studied by Chandrasekhar who worked out the requirement for the stability of a self-gravitating sphere. Solutions to the Einstein field equations admitting a polytropic equation of state are not common with physical viable solutions being a rarity [13]. This high- 
lights the nonlinearity of the Einstein field equations and the difficulty associated with solving them. Recently, researchers have been successful in modeling compact objects obeying a quadratic EoS of the form $p_{r}=\alpha \rho^{2}+\gamma \rho-\beta$ with $\alpha, \gamma$ and $\beta$ being constants [14-16]. Sharma and Ratanpal modeled anisotropic spheres by employing the Finch and Skea ansatz for one of the gravitational potentials. In order to close the system of equations they chose a physically plausible form for the radial pressure which facilitated the integration of the governing equation to admit the remaining gravitational potential. It is quite remarkable that such an approach leads to a class of physically viable solutions obeying a quadratic EoS [16]. A novel approach to constructing compact objects was used by Pant et al. in which they studied core-envelope models with the core obeying a linear EoS and the outer matter regions of the star described by a quadratic EoS. They show that such a composite configuration is stable and meets all the physical requirements for a physical realizable stellar structure [17]. They observed that an increase in mass leads to higher core density and more compact objects signifying the role played by gravity in the presence of larger masses.

By appealing to the microphysics associated with strange matter, Rocha et al. [5-23] adopt a CFL EoS of the form $p_{r}=\alpha \rho+\beta \rho^{\frac{1}{2}}-\gamma$ which generalises the MIT Bag model. Rocha et al. invoked the Durgapal and Banerjee transformation and a simple ansatz for one of the gravitational potentials to obtain an exact solution describing a compact object obeying a CFL EoS. In this study we model spherically symmetric compact objects by adopting an isotropic line element which is simultaneously comoving. The interior matter configuration is described by an anisotropic fluid with unequal stresses in the radial and tangential directions. The CFL EoS allows us to integrate the field equations thus reducing the problem to a single generating function of one of the gravitational potentials. By appealing to regularity, stability and physical viability of the gravitational and thermodyamical variables required for a realistic description of compact objects we present a tractable stellar model. We examine the behaviour of the CFL model in the linear approximation and show that the more robust linear EoS mimics the CFL EoS to a very good approximation.

\section{Spherical symmetry}

The interior spacetime of the stellar model takes the form

$d s^{2}=-A^{2}(r) d t^{2}+B^{2}(r)\left[d r^{2}+r^{2} d \Omega^{2}\right]$

where $d \Omega^{2}=d \theta^{2}+\sin ^{2} \theta d \phi^{2}$ and the metric functions, $A(r)$ and $B(r)$ are yet to be determined. In this paper we consider a model which represents a spherically symmetric, anisotropic fluid configuration. For our model the energymomentum tensor for the stellar fluid is

$T_{a b}=\operatorname{diag}\left(-\rho, p_{r}, p_{t}, p_{t}\right)$

where $\rho, p_{r}$ and $p_{t}$ are the proper energy density, radial pressure and tangential pressure respectively. The fluid fourvelocity $\mathbf{u}$ is comoving and is given by

$u^{a}=\frac{1}{A} \delta_{0}^{a}$

The Einstein field equations for the line element (1) are

$$
\begin{aligned}
& \rho=-\frac{1}{B^{2}}\left(2 \frac{B^{\prime \prime}}{B}-\frac{B^{\prime 2}}{B^{2}}+\frac{4}{r} \frac{B^{\prime}}{B}\right) \\
& p_{r}=\frac{1}{B^{2}}\left[\frac{B^{\prime 2}}{B^{2}}+2 \frac{A^{\prime}}{A} \frac{B^{\prime}}{B}+\frac{2}{r}\left(\frac{A^{\prime}}{A}+\frac{B^{\prime}}{B}\right)\right] \\
& p_{t}=\frac{1}{B^{2}}\left[\frac{A^{\prime \prime}}{A}+\frac{B^{\prime \prime}}{B}-\frac{B^{\prime 2}}{B^{2}}+\frac{1}{r}\left(\frac{A^{\prime}}{A}+\frac{B^{\prime}}{B}\right)\right]
\end{aligned}
$$

where primes represent differentiation with respect to the radial coordinate $r$. In generating the above field equations we have utilized geometrized units where the coupling constant and the speed of light are taken to be unity. The system (4)(6) comprises three independent equations with five variables $\left(A(r), B(r), \rho, p_{r}\right.$ and $\left.p_{t}\right)$. The mass contained within a radius $r$ of the sphere is defined as

$m(r)=\frac{1}{2} \int_{0}^{r} \omega^{2} \rho(\omega) d \omega$

For a physically realistic relativistic star we expect that the matter distribution should satisfy a barotropic equation of state $p_{r}=p_{r}(\rho)$. In this paper our object is to model an anisotropic compact sphere with CFL matter distribution. The EoS for CFL matter was well motivated by Rocha et al. [22] in which they assumed that the stellar interior was composed entirely of CFL matter. In order to make the problem mathematically tractable but without losing the essential physics at play, they were able to write the CFL EoS entirely as a function of the fluid density

$p_{r}=\frac{1}{3} \rho+\frac{2 \eta}{\pi} \rho^{1 / 2}-\left(\frac{3 \eta^{2}}{\pi^{2}}+\frac{4}{3} B\right)$

where

$\eta=-\frac{m_{s}^{2}}{6}+\frac{2 \Delta^{2}}{3}$

with $m_{s}$ being the quark mass, $B$ is the MIT Bag constant and $\Delta$ represents the quark interactions. In this regard we 
assume the EoS obeyed by our model takes the form

$p_{r}=\alpha \rho+\beta \rho^{\frac{1}{2}}-\gamma$

where $\alpha, \beta$ and $\gamma$ are real constants. The EoS of the form (10) has been used to model compact stars with CFL quark matter [22,23]. Substituting (4) and (5) into (10) we obtain the master equation

$$
\begin{aligned}
\frac{A^{\prime}}{A}= & -\left[2 \alpha \frac{B^{\prime \prime}}{B}+(1-\alpha) \frac{B^{\prime 2}}{B^{2}}+2(1+2 \alpha) \frac{1}{r} \frac{B^{\prime}}{B}\right. \\
& \left.+\gamma B^{2}+\beta B\left(-\frac{2 B^{\prime \prime}}{B}+\left(\frac{B^{\prime}}{B}\right)^{2}-\frac{4}{r} \frac{B^{\prime}}{B}\right)^{\frac{1}{2}}\right] \\
& \times\left[\frac{1}{2\left(\frac{1}{r}+\frac{B^{\prime}}{B}\right)}\right]
\end{aligned}
$$

The solution of which yields the gravitational behaviour of our model. On integrating (11) we obtain

$A=d \exp \left[-\frac{1}{2} \int F(r) d r\right]$

where

$$
\begin{aligned}
F(r)= & {\left[2 \alpha \frac{B^{\prime \prime}}{B}+(1-\alpha) \frac{B^{\prime 2}}{B^{2}}+2(1+2 \alpha) \frac{1}{r} \frac{B^{\prime}}{B}+\beta B^{2}\right.} \\
& \left.+\beta B\left(-\frac{2 B^{\prime \prime}}{B}+\left(\frac{B^{\prime}}{B}\right)^{2}-\frac{4 B^{\prime}}{r}\right)^{\frac{1}{2}}\right]\left(\frac{1}{\frac{1}{r}+\frac{B^{\prime}}{B}}\right)
\end{aligned}
$$

and $d$ is a constant of integration. Hence the line element (1) can be written as

$d s^{2}=-d^{2} \exp \left[-\int F(r) d r\right] d t^{2}+B^{2}\left[d r^{2}+r^{2} d \Omega^{2}\right]$

where $F(r)$ is given in (13). Hence, any solution describing static spherically symmetric anisotropic matter distribution with CFL equation of state in isotropic coordinate can be easily determined by a single generating function $B(r)$.

\section{Uniform density sphere}

If we assume that the density of the interior matter distribution is constant, $\rho=\mu=$ constant, (4) becomes

$\mu=-\frac{1}{B^{2}}\left(2 \frac{B^{\prime \prime}}{B}-\frac{B^{\prime 2}}{B^{2}}+\frac{4}{r} \frac{B^{\prime}}{B}\right)$
This equation admits the solution

$B=\frac{2 \sqrt{3} c}{\sqrt{\mu}\left(1+c^{2} r^{2}\right)}$

where $c$ is an arbitrary constant. This solution was first derived by Wyman in 1946 [24]. Substituting (16) in (11) we obtain

$A=d\left[\frac{1+c^{2} r^{2}}{1-c^{2} r^{2}}\right]^{\frac{\mu(1+3 \alpha)-3(\gamma+\beta \sqrt{\mu})}{2 \mu}}$

In this case the metric (1) take a particular form

$\begin{aligned} d s^{2}= & -d^{2}\left[\frac{1+c^{2} r^{2}}{1-c^{2} r^{2}}\right]^{\frac{\mu(1+3 \alpha)-3(\gamma+\beta \sqrt{\mu})}{\mu}} d t^{2} \\ & +\chi^{2}\left[d r^{2}+r^{2} d \Omega^{2}\right]\end{aligned}$

where $\chi^{2}=\frac{12 c^{2}}{\mu\left(1+c^{2} r^{2}\right)^{2}}$

\section{Compact stellar model}

If we set $B=\frac{a}{\sqrt{1+b r^{2}}}$, from (11) we obtain

$$
\begin{aligned}
A= & d\left(1+b r^{2}\right)^{\frac{(1+5 \alpha)}{4}}\left(\sqrt{1+b r^{2}}+\sqrt{6+b r^{2}}\right)^{-\frac{5 a \beta}{4 \sqrt{b}}} \\
& \times \exp \left[\left(b(1+\alpha)-a^{2} \gamma\right)\left(1+b r^{2}\right)\right. \\
& \left.-a \beta \sqrt{b} \sqrt{1+b r^{2}} \sqrt{6+b r^{2}}\left(\frac{1}{4 b}\right)\right]
\end{aligned}
$$

In this case the matter variables can be explicitly written as

$$
\begin{aligned}
\rho & =\frac{b\left(6+b r^{2}\right)}{a^{2}\left(1+b r^{2}\right)} \\
p_{r} & =\alpha \frac{b\left(6+b r^{2}\right)}{a^{2}\left(1+b r^{2}\right)}+\frac{\beta}{a} \sqrt{\frac{b\left(6+b r^{2}\right)}{\left(1+b r^{2}\right)}}-\gamma \\
p_{t} & =\frac{\left(1+b r^{2}\right)}{a^{2}}\left[\frac{A^{\prime \prime}}{A}+\frac{A^{\prime}}{r A}-\frac{2 b}{\left(1+b r^{2}\right)^{2}}\right] \\
\Delta & =p_{t}-p_{r}
\end{aligned}
$$

where $\Delta=p_{t}-p_{r}$ is the measure of anisotropy. It is noted that the matter variables are given in terms of elementary functions that facilitate for a detailed physical analysis of the model. In this case the (28) takes form

$m(r)=\frac{b r^{3}+15\left(r-\frac{\arctan [\sqrt{b} r]}{\sqrt{b}}\right)}{6 a^{2}}$ 


\section{Physical analysis}

We are now in a position to discuss the physical features of our model. It is noted that the model generated should satisfy the following physical requirements to describe a realistic star:

(i) regularity of the gravitational potentials at the origin;

(ii) positive definiteness of the energy density and the radial pressure at the origin;

(iii) vanishing of the pressure at some finite radius;

(iv) monotonic decrease of the energy density, the radial pressure and tangential pressure with increasing radius;

(v) interior metric match smoothly with the exterior metric:

$$
d s^{2}=-\frac{\left(1-\frac{M}{2 r}\right)^{2}}{\left(1+\frac{M}{2 r}\right)^{2}} d t^{2}+\left(1+\frac{M}{2 r}\right)^{4}\left[d r^{2}+r^{2} d \Omega^{2}\right]
$$

across the boundary $r=R$, where $M$ is the total mass of the sphere;

(vi) casuality condition: both the radial and tangential speed of sound is less than the speed of light throughout the interior of star i.e., $0 \leq V_{r}^{2}=\frac{d p_{r}}{d \rho} \leq 1$ and $0 \leq V_{t}^{2}=\frac{d p_{t}}{d \rho} \leq 1$

(vii) satisfy the stability condition: $-1 \leq V_{t}^{2}-V_{r}^{2} \leq 0$;

(viii) a physically reasonable energy-momentum tensor has to obey the conditions $\rho-p_{r}-2 p_{t} \geq 0$ and $\rho+$ $p_{r}+2 p_{t} \geq 0$

(ix) ratio of trace of stress tensor to density, $\frac{p_{r}+2 p_{t}}{\rho}$, should decrease from centre to the surface of star.

Now we show that the models generated in Sect. 4 satisfy the above listed physical properties.

(i) Since $A^{2}(0)=d^{2}(1+\sqrt{6})^{\frac{-5 \alpha \beta}{2 \sqrt{b}}} \exp \left[\frac{b(1+\alpha)-a^{2} \gamma-\alpha \beta \sqrt{6 b}}{2 b}\right]$, $B^{2}(0)=a^{2}$ which are constants and $\left(A^{2}(r)\right)^{\prime}=$ $\left(B^{2}(r)\right)^{\prime}=0$ at the origin $r=0$, the gravitational potentials are regular at the origin.

(ii) Since $\rho(0)=\frac{6 b}{a^{2}}$ and $p_{r}(0)=\frac{6 \alpha b}{a^{2}}+\frac{\beta \sqrt{6 b}}{a}-\gamma$, the energy density and radial pressure are positive at the origin.

(iii) At the boundary of the star $r=R$ the condition $p_{r}(r=$ $R)=0$ impose the restriction $\alpha \frac{b\left(6+b r^{2}\right)}{a^{2}\left(1+b r^{2}\right)}+\frac{\beta}{a} \sqrt{\frac{b\left(6+b r^{2}\right)}{\left(1+b r^{2}\right)}}-$ $\gamma=0$ on the parameters $a, b, \alpha, \beta$ and $\gamma$.

(iv) Since $\frac{d \rho}{d r}=-\frac{10 b^{2} r}{a^{2}\left(1+b r^{2}\right)^{2}}<0$, the energy density is a decreasing function of $r$. The radial pressure $p_{r}$ is also a decreasing function as $p_{r}$ and $\rho$ are related on a linear EoS.

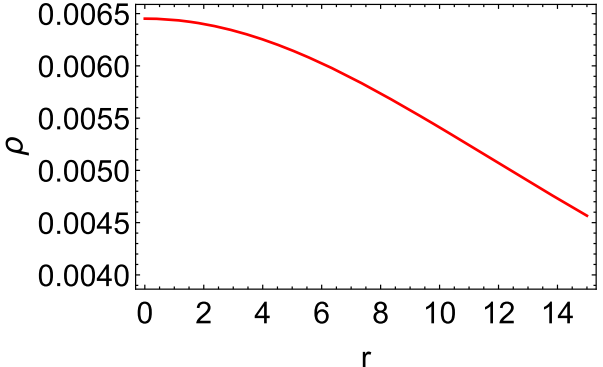

Fig. 1 Variation of density with radial coordinate

(v) Matching of interior metric (1) and exterior metric (25) at the boundary $r=R$ leads to the constrains

$$
\begin{aligned}
& A(R)=\frac{\left(1-\frac{M}{2 R}\right)}{\left(1+\frac{M}{2 R}\right)} \\
& B(R)=\left(1+\frac{M}{2 R}\right)^{2}
\end{aligned}
$$

where

$M=m(R)=\frac{b R^{3}+15\left(R-\frac{\arctan [\sqrt{b} R]}{\sqrt{b}}\right)}{6 a^{2}}$

The condition (26) imposes the following restriction on the constant of integration

$$
\begin{aligned}
d= & \frac{12 a^{2} R-\left[b R^{3}+15\left(R-\frac{\arctan [\sqrt{b} R]}{\sqrt{b}}\right)\right]}{12 a^{2} R+\left[b R^{3}+15\left(R-\frac{\arctan [\sqrt{b} R]}{\sqrt{b}}\right)\right]} \\
& \times\left[1+b R^{2}\right]^{-\frac{(1+5 \alpha)}{4}} \\
& \times \exp \left[-\frac{\left[b(1+\alpha)-a^{2} \beta\right]\left(1+b R^{2}\right)}{4 b}\right]
\end{aligned}
$$

The condition (27) implies

$$
\frac{a}{\sqrt{1+b r^{2}}}=\left[1+\frac{b R^{3}+15\left(R-\frac{\arctan [\sqrt{b} R]}{\sqrt{b}}\right)}{12 a^{2} R}\right]^{2}
$$

These impose a restriction on the parameters $a, b$ and can be solved for one of the parameter if the values for the radius of the sphere and the remaining parameter are specified.

(vi) The surface redshift $Z s=\left(1-\frac{2 M}{R}\right)^{-1 / 2}-1$ [28] decreases with increasing radius of the sphere. 


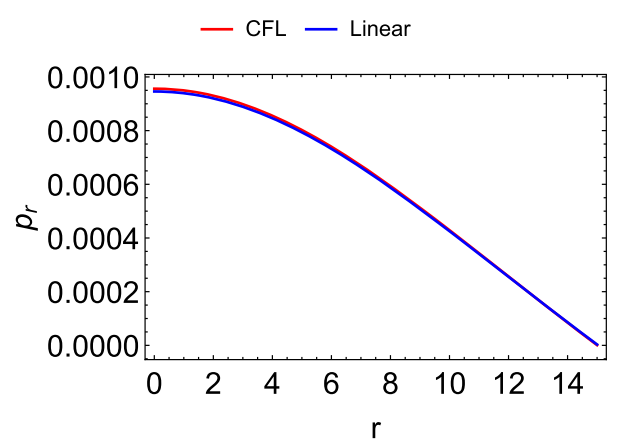

Fig. 2 Variation of radial pressure with radial coordinate

Due to the complexity of the solution, we show graphically that the matter variables are well behaved throughout the interior of the star. The figures were plotted in a way to compare the effect of CFL matter EoS with linear EoS. To plot the figures for both CFL matter and linear EoSs we fixed the values for the parameters $a=1.49399, b=0.0024, \alpha=0.5$ and $\beta=0.001, \gamma=0.00235$ for CFL matter EoS and $\beta=0, \gamma=0.00228$ for linear EoS. From Fig. 1 we observe that the energy density is a monotonically decreasing function of the radial coordinate. In our construction of our stellar model the energy density is the same in both the CFL and linear regimes. The radial pressure is displayed in Fig. 2. As expected the pressure drops off monotonically from the center of the star and vanishes for some finite value of the radial coordinate. The radial pressures for both the CFL and linear models are indistinguishable at each interior point of the fluid configuration. The tangential pressure is plotted in Fig. 3. We observe that the tangential pressure decreases monotonically outwards towards the boundary of the star with the linear EoS pressure dominating its CFL counterpart throughout the stellar interior. We observe an interesting behaviour of the anisotropy parameter in Fig. 4. While $\Delta \geq 0$ throughout the interior for the linear EoS, we note that the anisotropy parameter changes sign within the CFL configuration. This implies that force associated with anisotropy can be repulsive $\left(p_{t}>p_{r}\right)$ or attractive $\left(p_{t}<p_{r}\right)$. Figure 4 shows that the central regions experience an attractive force due to pressure anisotropy making these regions more unstable. As one moves outwards towards the surface layers of the star, the anisotropy changes sign, becoming positive. The surface layers of the object are subjected to a repulsive force due to pressure anisotropy thus making this region more stable against the inwardly acting gravitational attraction. Figures 5 and 6 show the variation of the square of the radial and tangential speeds respectively. These quantities play an important role in determining stable and unstable regions within the object as proposed by Herrera [25]. According to Abreu et al. the condition for stable regions to exist within the core, we must have $-1<V_{t}^{2}-V_{r}^{2} \leq 0$ [26]. Figure 7 shows that the star is composed of stable regions as one moves from the centre

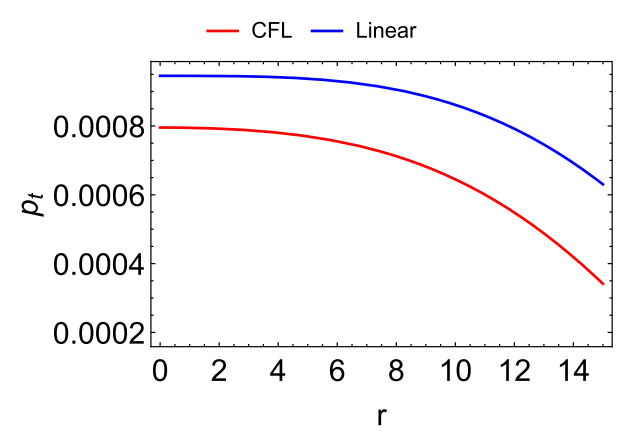

Fig. 3 Variation of tangential pressure with radial coordinate

outwards towards the boundary. In addition, the linear EoS predicts more stable regions than the CFL EoS. It is well known that the ratio of the specific heats for an anisotropic fluid is given by

$\Gamma<\frac{4}{3}-\left[\frac{4}{3} \frac{p_{r}-p_{t}}{\left|p_{r}^{\prime}\right| r}\right]_{\max }$

We note that the anisotropy increases the instability of the collapsing system when $p_{r}<p_{t}$. We obtain the classical Newtonian result, $\Gamma<\frac{4}{3}$ in the case of isotropic pressure, $p_{r}=p_{t}$ as a measure of the instability of the stellar configuration. Figure 8 shows that $\Gamma_{r}>\frac{4}{3}$ throughout the fluid configuration indicative of a stable object. As pointed out in Fig. 4, the anisotropy changes sign in different regions of the configuration. The change in sign of the anisotropic factor is not sufficiently large to render the configuration unstable as supported by the trend in Fig. 8. We also observe that the adiabatic stability index is indistinguishable for both the linear and CFL EoS's. For the anisotropic fluid configurations, the strong energy condition $\rho-p_{r}-2 p_{t} \geq 0$ has to be satisfied within the stellar interior. Figures 9 and 10 show that the weak and strong energy conditions are satisfied throughout the matter distribution. It has been shown that the generated model satisfy all the physical requirements listed above to represent a realistic star in both CFL and linear EoS's. The mass profile is displayed in Fig. 11. We observe that the mass vanishes at the centre of the configuration and increases as the radial coordinate increases. In Fig. 12 we have plotted the surface redshift as a function of the proper radius. The surface redshift decreases as the radius increases. This trend is in agreement with findings reported by Zhao and Jia [27] who studied surface redshifts of neutron stars.

\section{Fixing parameters}

In this section we turn our attention to fixing the free parameters in our model. In order to obtain the radius of the star, we require the vanishing of the radial pressure for some finite 


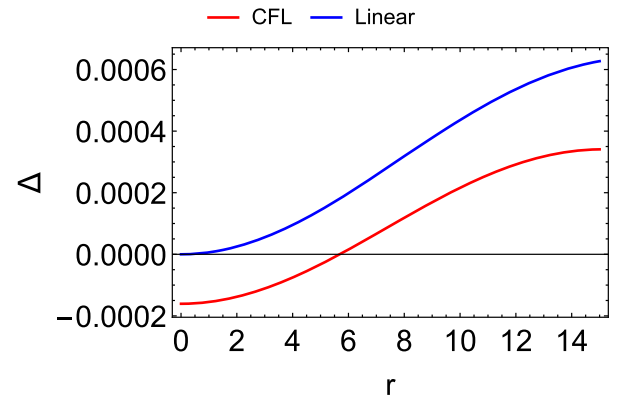

Fig. 4 Variation of anisotropy with radial coordinate

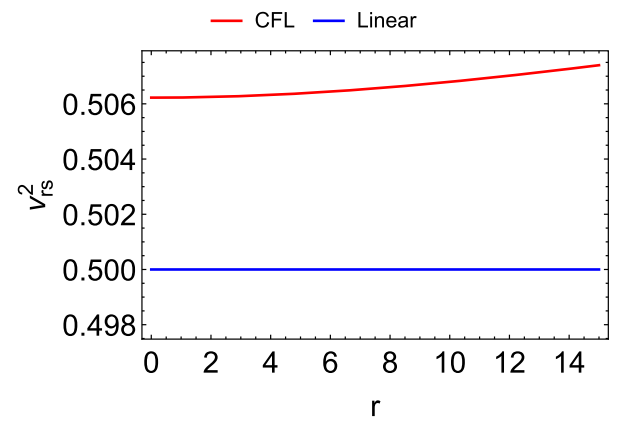

Fig. 5 Variation of radial speed with radial coordinate

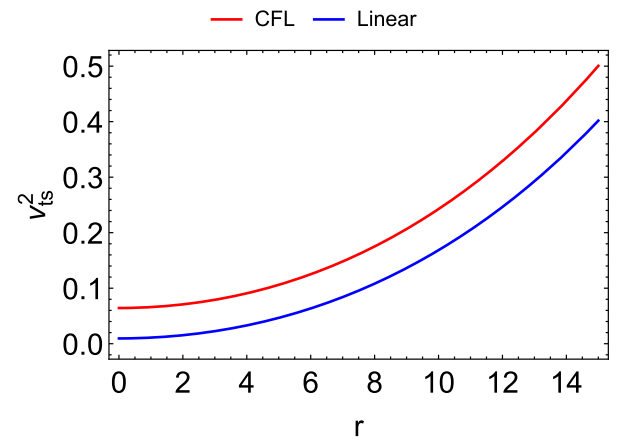

Fig. 6 Variation of tangential speed with radial coordinate

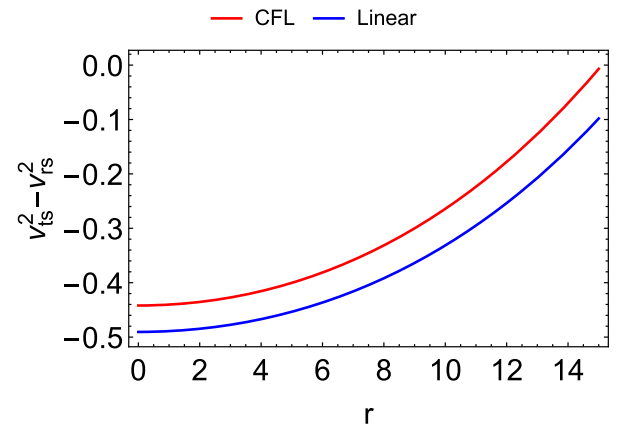

Fig. 7 Variation of stability with radial coordinate

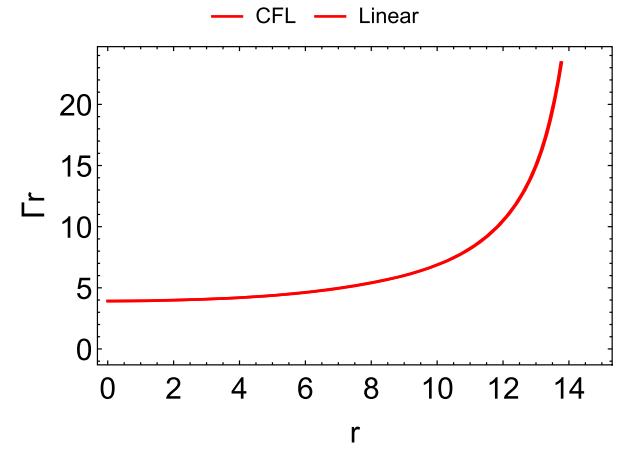

Fig. 8 Variation of $\Gamma_{r}$ with radial coordinate

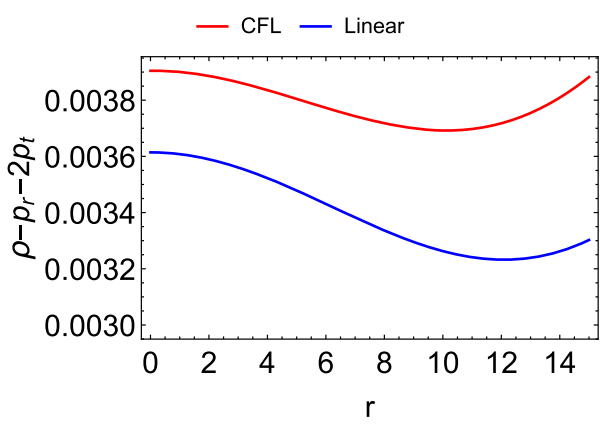

Fig. 9 Variation of weak energy condition as a function of the radial coordinate

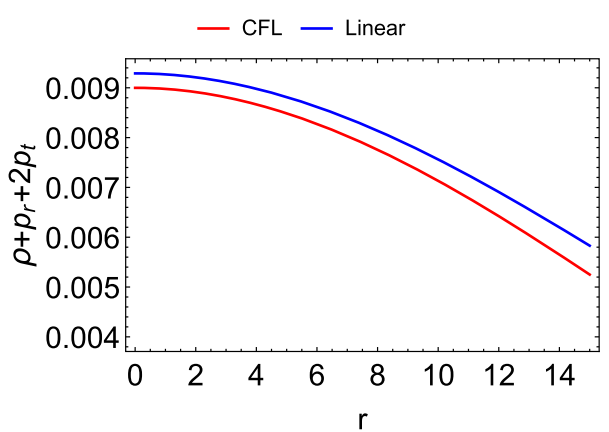

Fig. 10 Variation of the strong energy condition as a function of the radial coordinate

$r=R$. This requirement yields

$R_{c f l}=\sqrt{\frac{-12 b^{3}-18 a^{4} b \gamma^{2}+21 b^{2} \psi+15 \sqrt{3} \sqrt{\zeta}}{b^{2}\left(b^{2}-3 b \psi+9 a^{4} \gamma^{2}\right)}}$

where we have defined $\psi=3 \beta^{2}+2 a^{2} \gamma$ and $\zeta=b^{4} \beta^{2}(\psi+$ $2 a^{2} \gamma$ ). For the linear EoS the radius is given by

$R_{l}=\sqrt{3} \sqrt{\frac{2 b-a^{2} \gamma}{-b^{2}+3 a^{2} b \gamma}}$ 


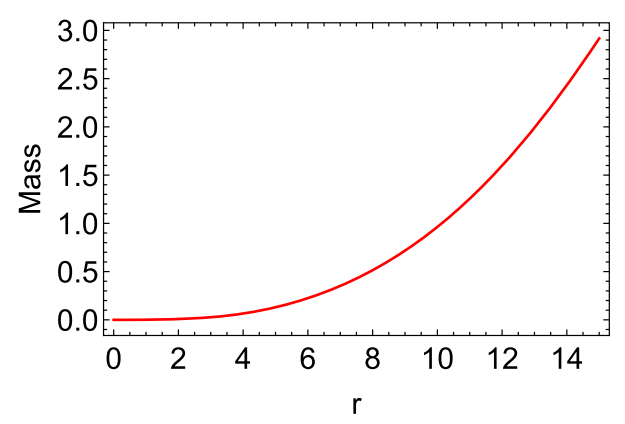

Fig. 11 Variation of mass with radial coordinate

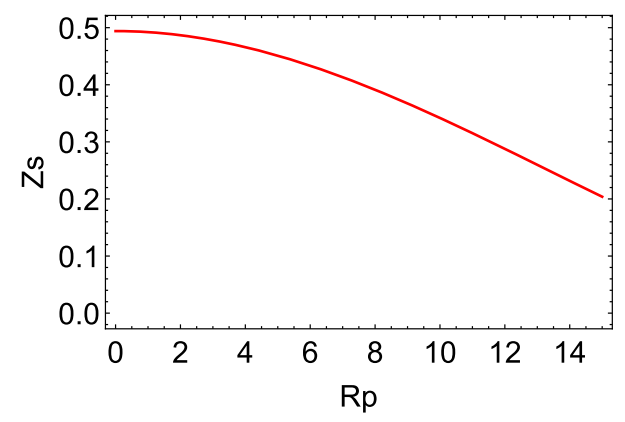

Fig. 12 Variation of surface redshift with radial coordinate

From (32) we arrive at the following restrictions

$$
\frac{a^{2} \gamma}{2} \leq b<3 a^{2} \gamma
$$

We further note from (20) that the constant $b$ can be written in terms of the central density, $\rho_{c}$ as

$b=\frac{a^{2} \rho_{c}}{6}$

and in the light of (33) we have

$3 \gamma<\rho_{c}<18 \gamma$

In order to plot $M$ vs $R$, we use (28) which depends on $(a, b, R)$. There are various ways to fit our model to observational data. If we choose the central density to be some multiple of the nuclear saturation density, then we can write $b$ in terms of $a$, with $\gamma$ being fixed in the process. The difficulty in this approach is that the effect of the vacuum term in the variation of the mass is lost. A second approach due to Rocha et al. [22] is to choose various values for the quark mass, $m_{s}$ and the gap term, $\Delta$, representing the quark interactions while keeping the MIT Bag constant fixed. This generates different values of $\gamma$. From (34) we are able to obtain different values for $b$ in terms of $a$ which encodes the contribution from the microphysics. This facilitates comparison between different models which are distinguished by the quark interactions in the stellar fluid. At this point we should point that the constant, $a$ is still free. This implies that there is sufficient freedom in our model to reconcile with observational data. We can conclude that it is always possible to fit characteristics of our model with observations because of the numerous degrees of freedom. On the flip side, physical characteristics of compact objects such as masses and radii depend on the observational techniques employed [29]. At best we can claim that these are toy models which allow us to get insights into the behaviour of the thermodynamical and gravitational behaviour of compact objects as evidenced in Figs. 1, 2, 3, $4,5,6,7,8,9,10,11$ and 12 . As to the fixing of the model parameters to observational data we must err on the side of caution and must distinguish between measured and inferred values.

\section{Conclusion}

This study focused on the comparison between the CFL and linear EoS exact solutions for compact stars consisting of the strange matter phase and allowing anisotropy in the pressure. The CFL phase is modeled as a charge free and colourless gas of quark Cooper pairs that allows this matter to be the true ground state. There is a large number of works, as those referenced in [1], that propose different classes of solutions for the Einstein field equations using toy models. The feature of our work is to provide a realistic linear EoS for the compact object, thus connecting micro and macrophysics and finding exact descriptions. In summary, we have provided two anisotropic pressure models made of CFL strange matter. This was achieved by obtaining a master equation from the CFL EoS by substituting for density and radial pressure. The following ansatz for one of the gravitational potentials, $B=\frac{a}{\sqrt{1+b r^{2}}}$ was used to obtain the other potential which then completes the gravitational behaviour of the model. The field equations satisfy all the physical requirements for a realistic star. By making $\beta=0$ the CFL EoS becomes a linear EoS thus allowing us to obtain a new set of field equations. By plotting the various thermodynamical quantities, stability criteria and energy conditions we show that the linear EoS gives a reasonable approximation to the CFL EoS.

Acknowledgements MG acknowledges the support from the office of the DVC: Research, Innovation and Engagement, Durban University of Technology and the National Research Foundation, South Africa.

Data Availability Statement This manuscript has no associated data or the data will not be deposited. [Authors' comment: There is no data to be deposited.]

Open Access This article is licensed under a Creative Commons Attribution 4.0 International License, which permits use, sharing, adaptation, distribution and reproduction in any medium or format, as long as you give appropriate credit to the original author(s) and the source, provide a link to the Creative Commons licence, and indicate if changes 
were made. The images or other third party material in this article are included in the article's Creative Commons licence, unless indicated otherwise in a credit line to the material. If material is not included in the article's Creative Commons licence and your intended use is not permitted by statutory regulation or exceeds the permitted use, you will need to obtain permission directly from the copyright holder. To view a copy of this licence, visit http://creativecomm ons.org/licenses/by/4.0/.

Funded by SCOAP ${ }^{3}$.

\section{References}

1. M.R.S. Delgaty, K. Lake, Comput. Phys. Commun. 115, 395-415 (1998)

2. R. Sharma, S.D. Maharaj, Mon. Not. R. Astron. Soc. 375, 1265 1268 (2007)

3. P.M. Takisa, S.D. Maharaj, S. Ray, Astrophys. Sp. Sci. 354, 463 (2014)

4. S.A. Ngubelanga, S.D. Maharaj, S. Ray, Astrophys. Sp. Sci. 357, 40 (2015)

5. K. Rajagopal, F. Wilczek, M.W. Paris, S. Reddy, Phys. Rev. Lett. 86, $3492(2001)$

6. O.G. Benvenuto, J.E. Horvath, Mon. Not. R. Astron. Soc. 241, 43-50 (1989)

7. F. Rahaman, R. Sharma, S. Ray, Eur. Phys. J. C. 72, 2071 (2012)

8. S. Biswas, S. Ghosh, B.K. Guha, F. Rahaman, S. Ray, Ann. Phys. 401, 1 (2019)

9. F.C. Ragel, S. Thirukkanesh, Eur. Phys. J. C 79, 306 (2019)

10. R. Sharma, S. Das, M. Govender and D. M. Pandya, Ann. Phys. (in press) (2020)
11. M. Dey, I. Bombaci, J. Dey, S. Ray, B.C. Samanta, Phys. Lett. B. 438, 123-128 (1998)

12. M. Kalam, A.A. Usmani, F. Rahaman, SkM Hossein, I. Karar, R. Sharma, Int. J. Theor. Phys. 52, 3319 (2013)

13. S.A. Ngubelanga, S.D. Maharaj, Eur. Phys. J. Plus. 130, 211 (2015)

14. T. Feroze, A.A. Siddiqui, Gen. Relativ. Gravit. 43, 1025 (2011)

15. S.A. Ngubelanga, S.D. Maharaj, S. Ray, Astrophys. Sp. Sci. 357, 74 (2015)

16. R. Sharma, B.S. Ratanpal, Int. J. Mod. Phys. D 22, 1350074 (2013)

17. N. Pant, S. Gedela, R.P. Pant, J. Upreti and R.K. Bisht, Eur. Phys. J. Plus, https://doi.org/10.1140/epjp/s13360-020-00209 (2020)

18. P. Bhar, M. Govender, R. Sharma, Eur. Phys. J. C 77, 8 (2017)

19. M. Alford, K. Rajagopal, S. Reddy, F. Wilczek, Phys. Rev. D 64, 074017 (2001)

20. G. Lugones, J.E. Horvath, Phys. Rev. D 66 (2002)

21. G. Lugones, J.E. Horvath, Astr. Astrophys. 403, 173 (2003)

22. L.S. Rocha, A. Bernardo, M.G.B. de Avellar, J.E. Horvath, Astron. Nachr. 340, 180-183 (2019)

23. Rocha et al., arXiv:1906.11311v2 gr-qc (2019)

24. M. Wyman, Phys. Rev. D. 70, 74 (1946)

25. L. Herrera, J. Ospino, A. Di Prisco, Phys. Rev. D 77, 027502 (2008)

26. H. Abreu et al., Class. Quant. Grav. 24, 4631 (2007)

27. X.-F. Zhao, H.-Y. Jia, Revista Mexicana de Astronomia y Astrofisica. 50, 103 (2014)

28. R.L. Bowers, E.P.T. Liang, Astrophys. J. 188, 657 (1974)

29. J.M. Lattimer, Universe 5, 159 (2019) 